\title{
Prediction in Quantum Cosmology
}

\author{
James B. Hartle \\ Department of Physics \\ University of California \\ Santa Barbara, CA 93106, USA
}

\begin{abstract}
Lectures at the 1986 Cargèse summer school publshed in Gravitation in Astrophysics ed. by J.B. Hartle and B. Carter, Plenum Press, New York (1987). In this version some references that were 'to be published' in the original have been supplied, a few typos corrected, but the text is only modestly edited. The author's views on the quantum mechanics of cosmology have changed in important ways from those presented in Section 2. (See, e.g. J.B. Hartle, Spacetime Quantum Mechanics and the Quantum Mechanics of Spacetime in Gravitation and Quantizations, Proceedings of the 1992 Les Houches Summer School, ed. by B. Julia and J. Zinn-Justin, Les Houches Summer School Proceedings Vol. LVII, North Holland, Amsterdam (1995). The material in Sections 3 and 4 on the classical geometry limit and the approximation of quantum field theory in curved spacetime may still be of use.
\end{abstract}

\section{Introduction}

As far as we know them, the fundamental laws of physics are quantum mechanical in nature. If these laws apply to the universe as a whole, then there must be a description of the universe in quantum mechancal terms. Even our present cosmological observations require such a description in principle, though in practice these observations are so limited and crude that the approximation of classical physics is entirely adequate. In the early universe, however, the classical approximation is unlikely to be valid. There, towards the big bang singularity, at curvatures characterized by the Planck length, $\left(\hbar G / c^{3}\right)^{\frac{1}{2}}$, quantum fluctuations become important and eventually dominant.

Were the aim of cosmology only to describe the present universe, expressing that description in quantum mechanical terms might be an interesting intellectual exercise but of no observational relevance. Today, however, we have a more ambitious aim: to explain the presently observed universe by a simple and compelling laws of its quantum state and dynamics. It is natural to expect such laws to be quantum mechanical for several reasons: The laws must describe the early universe where quantum gravitational fluctuations are important. In quantum fluctuations we can imagine a simple origin of present complexity. Finally, if all the other fundamental laws of physics are quantum mechanical, it is only natural to expect the laws of initial conditions and dynamics to 
be so also. It is for the search for these that we need a quantum mechanical description of the universe - a quantum cosmology $[1,2-5]$. The nature of this description is the subject of these lectures [6].

In the application of quantum mechanics to the universe as a whole, one confronts the characteristic features of quantum theory in a striking and unavoidable manner. Some find these features uncomfortable, or unsatisfactory, or even absurd. It is not the purpose of these lectures to examine whether these attitudes represent a success or failure of intuition. Rather, the purpose is to sketch how the standard quantum theory, or a suitable generalization of it, can be used to frame a law of initial conditions and to extract from it predictions for cosmological observation. We shall thus assume quantum mechanics.

We shall also assume spacetime. While it is uncertain whether Einstein's vision of spacetime as a fundamental dynamical quantity is correct, it is perhaps the most compelling viewpoint in which to frame a quantum theory of cosmology. Within this framework of quantum spacetime our discussion will, in large part, be general and not single out any particular dynamics or particular theory of initial conditions.

The aim of these lectures is to show how the single system which is our universe is described in a quantum theory of spacetime and to sketch how a prescription for the quantum state of the universe can be used to make verifiable predictions. This is discussed in general in Sections 2 and 3. However, as the observations which are accessible to us are describable in classical terms, extracting predictions in the classical limit is a particular problem of special importance. This is considered in Section 4. As an interesting by product of this discussion, we describe the connection between a quantum theory of spacetime and the approximation of quantum field theory in curved spacetime.

\section{Predictions of the Wave Function of the Universe}

\subsection{The Wave Function of the Universe}

In quantum mechanics we describe the state of a system by giving its wave function. The wave function enables us to made predictions about observations made on a spacelike surface; it thus captures quantum mechanically the classical notion of the "state of the system at a moment of time." The arguments of the wave function are the variables describing how the system's history intersects the spacelike surface. For example, for the quantum mechanics of a particle, the histories are particle paths $x(t)$. We write for the wave function

$$
\psi=\psi(x, t) .
$$

The $t$ labels the hypersurface and the $x$ specifies the intersection of the history with it.

In the quantum mechanics of a closed cosmologies with fixed (for simplicity) spatial topology, say that of a 3 -sphere $S^{3}$, the histories are the 4-geometries, ${ }^{4} \mathcal{G}$, on $\mathbf{R} \times S^{3}$. The appropriate notion of a 4-geometry fixed on a spacelike surface is the 3-geometry,

${ }^{3} \mathcal{G}$, induced on that surface. One can think of this as specified by a 3 -metric $h_{i j}(\mathbf{x})$ on the fixed spatial topology. Thus for the quantum mechanics of a closed cosmology we 
write [7]

$$
\Psi=\Psi\left[{ }^{3} \mathcal{G}\right]=\Psi\left[h_{i j}(\mathbf{x})\right] .
$$

Note that there is no additional "time" label. This is because a generic 3-geometry will fit into a generic 4-geometry at locally only one place if it fits at all. The 3-geometry itself carries the information about its location in spacetime. For example, the 4-geometry of a closed Friedman universe is described by the metric

$$
d s^{2}=-d \tau^{2}+a^{2}(\tau) d \Omega_{3}^{2},
$$

where $d \Omega_{3}^{2}$ is the metric on the round 3-sphere. A 3-geometry is described by the radius of a 3-sphere. This radius locates us locally in the spacetime although in the large there are two values of $\tau$ for each value of $a$. This labeling of the wave function correctly counts the degrees of freedom. Of the six components of $h_{i j}$, three are gauge. If one of the remaining three is time, there are left two degrees of freedom - the correct number for the massless, spin-2 gravitational field.

The space of all three geometries is called superspace. Each "point" represents a different geometry on the fixed spatial topology. In the case of pure gravity that we have been describing, the wave function is a complex function on superspace. With the inclusion of matter fields the wave function depends on their configurations on the spacelike surface as well, and we write typically

$$
\Psi=\Psi\left[h_{i j}(\mathbf{x}), \Phi(\mathbf{x})\right]
$$

A law for initial conditions in quantum cosmology is a law which prescribes this wave function.

\subsection{Cosmological Observations and Cosmological Predictions}

To make contact with observations we must specifiy the observational consequences of the state of the universe being described by this or another wave function. This is usually called an "interpretation" of $\Psi$. There is little doubt that what I can say here will not address every issue which can be raised on this fascinating topic and even less doubt that it will not satisfy many who have thought about the subject. I would like, however, to offer some minimal elements of an interpretation which I believe will enable an attribution of $\Psi$ to the universe to be confronted by cosmological observations. These elements are an example of "an Everett interpretation" although the words and emphasis may be different from other interpretations in this broad catagory [8].

There are at least three problems to be addressed (1) the special nature of cosmological predictions, (2) the quantum mechanics of single systems, and (3) the problem of time. We shall discuss them in order.

\subsection{The Nature of Cosmological Predictions}

The favorite paradigm of prediction in physics is evolution: If we start the system in a certain state then a time $t$ later we predict that it will be in a certain other state. This type of problem conforms to the characteristic form of predictive statements. If "this" 
then "that" - a correlation between experimental conditions and observations. In placing conditions and observations in temporal order, however, it is very uncharacteristic of predictions we can make in the astrophysical and geological sciences. In geology we might predict as follows: "If we are in a certain type of strata, then we should find a certain type of dinosaur bone." "If we are in the middle of an ocean floor, then we predict an upwelling trench" and so on. Here condition and observation are at the same time. This, prediction of correlations at the same time is, I believe, characteristic of systems over which we have little experimental control.

Cosmology is much the same. We can, of course, imagine a $10^{9}$ year experiment "Given the observations of the positions of the galaxies now, we predict that if we wait

$10^{9}$ years, we will see them in new positions..." Such a prediction is a test of a theory of initial conditions because the longer we wait the more initial data we see. It is, however, not very practical and therefore not very interesting.

A more interesting type of prediction is a $10^{9}$ franc experiment: Suppose you are allocated $10^{9}$ francs to build new optical, radio, X-Ray, neutrino and gravitational wave telescopes, what do you predict you will see? A typical prediction might be the following: "Given the locally measured values of the Hubble constant and the mass density, we predict that at great distances we will see the same uniform mass density, a certain galaxy-galaxy correlation function, a certain gravitational wave background, etc." Characteristically these predictions involve conditions and observations at a single moment of time.

\subsection{Quantum Mechanics of Individual Systems}

The idea for dealing with the universe as a single system is to take quantum mechanics seriously. One assumes that there is one wave function $\Psi$ defined on a preferred configuation space which contains all the predictable information about observations made on a spacelike surface. If $\Psi$ is sufficiently peaked about some region in the configuration space, we predict that we will observe the correlations between the observables which characterize this region. If $\Psi$ is small in some region, we predict that observations of the correlations which characterize this region are precluded. Where $\Psi$ is neither small nor sufficiently peaked we don't predict anything. That's it.

The natural reaction to such a proposal for interpretation is to ask "Where is probability?" In response, two things can be said. First, probabilities for single systems have no direct observational meaning and the universe, by definition, is a single system. Second, as we shall show below, this interpretation implies the usual probability interpretation of ordinary quantum mechanics when applied to ensembles of identically prepared systems.

In cosmology, therefore, we would examine any particular proposal for $\Psi$ to see which correlations are predicted - those on which the wave function is sharply peaked, and which are precluded - those on which it is essentially zero. We would ask, for example: "Given the value of the Hubble constant and the local mean density is the wave function sharply peaked about a form of the galaxy-galaxy correlation function?" If so we predict that correlation of variables. Note that characteristically we have conditions and observations on a single spacelike surface. This type of interpretation means that 
one's ability to predict in quantum cosmology is very limited. Given a value of the Hubble constant and the local mean mass density, one can ask whether the wave function sharply peaked about the number of planets in the solar system, or the architecture of this building, or the weights of the participants of this conference. I, for one, hope not. One of the central problems in quantum cosmology is therefore to find what correlations are predicted and how specific must we be in conditions to get predictions for interesting observations. (Problem 1).

Ordinary quantum mechanics can be formulated as a theory of individual systems. Indeed, a moments reflection will show that this has to be so. Quantum mechanics formulated only in terms of probabilities would make definite predictions only about infinite ensembles - an idealization we do not encounter in the real world. Any ensemble can be regarded as a single system composed of many identical parts. Quantum mechanics should be formulable as a theory of individual systems and the probability interpretation derivable from the predictions this formulation makes about single systems with many identical subsystems. In the late '60's a number of workers independently showed how to do this [9]:

Consider a single system and let it be described by a wave function $\psi$. Possible observations correspond to operators in the Hilbert space of states. For the physical interpretation of $\psi$ for an individual system assume only the following: If $\psi$ is an eigenfunction of an observable $A$ then an observation of $A$ will yield the eigenvalue. For those observables of which $\psi$ is not an eigenfunction there is no prediction for the outcome of an observation. We can then derive the probability interpretation of $\psi$ as follows:

Suppose the configuration space of the single system is $C$; the configuration space for an ensemble of $N$ systems is $C^{N}$. An ensemble of $N$ systems each in the identical state $\psi(q)$ is described by the wave function on $C^{N}$

$$
\Psi\left(q_{1}, \cdots, q_{n}\right)=\psi\left(q_{1}\right) \psi\left(q_{2}\right) \cdots \psi\left(q_{n}\right)
$$

On the Hilbert space of wave functions on $C^{N}$ there is an operator $\hat{f}_{a}$ corresponding to observing $q$ on the first system, $q$ on the second, etc., and then computing the frequency that a given value $a$ occurs. For an infinitely large ensemble of identical systems, each in a state $\psi$, is it a mathematical fact that the product wave function (2.5) is an eigenfunction of this operator

$$
\hat{f}_{a} \Psi=|\psi(a)|^{2} \Psi .
$$

The predicted frequency is the square of the wave function of the single system. In this way we deduce the probability interpretation of quantum mechanics from its predictions about individual systems.

To see how this works let us consider a definite example. Consider ensembles of spin $-1 / 2$ systems. A single system has states $\mid S>, S=\uparrow$ or $\downarrow$, and the Hilbert space of an ensemble of $N$ systems is spanned by the basis

$$
\left|S_{1}>\right| S_{2}>\cdots \mid S_{N}>
$$

In this basis we can define the operator corresponding to a measurement of the relative 
frequency of say spin up, $\uparrow$

$$
f_{\uparrow}^{N}=\sum_{S_{1} \cdots S_{N}}\left|S_{1}>\cdots\right| S_{N}>\left(\sum_{S_{i}} \frac{\delta_{S_{i} \uparrow}}{N}\right)<S_{N}\left|\cdots<S_{1}\right| .
$$

Consider now the expectation value of $f_{\uparrow}^{N}$ in the state of an ensemble of identically prepared systems each in state $|\psi\rangle$

$$
\left|\psi^{N}>=\right| \psi>|\psi>\cdots| \psi>\text {. }
$$

It is

$$
\begin{aligned}
<\psi^{N}\left|f_{\uparrow}^{N}\right| \psi^{N}> & =\sum_{S_{1} \cdots S_{N}}\left(\sum_{S_{i}} \frac{\delta_{\uparrow S_{i}}}{N}\right)\left|<S_{1}\right| \psi>\left.\right|^{2} \cdots\left|<S_{N}\right| \psi>\left.\right|^{2} \\
& =|<\uparrow| \psi>\left.\right|^{2} \sum_{S_{2} \cdots S_{N}}\left|<S_{2}\right| \psi>\left.\right|^{2} \cdots\left|<S_{N}\right| \psi>\left.\right|^{2}=|<\uparrow| \psi>\left.\right|^{2} .
\end{aligned}
$$

Consider also the fluctuations about this mean value:

$$
<\psi^{N}\left|\left(f_{\uparrow}^{N}-|<\uparrow| \psi>\left.\right|^{2}\right)^{2}\right| \psi^{N}>=<\psi^{N}\left|\left(f_{\uparrow}^{N}\right)^{2}\right| \psi^{N}>-|<\uparrow| \psi>\left.\right|^{4} .
$$

The first term is

$$
\begin{gathered}
\sum_{S_{1} \cdots S_{N}}\left(\sum_{i=j} \frac{\delta_{\uparrow S_{i}}}{N^{2}}+\sum_{i \neq j} \frac{\delta_{\uparrow S_{i}} S_{\uparrow S_{j}}}{N^{2}}\right)\left|<S_{1}\right| \psi>\left.\right|^{2} \cdots\left|<S_{N}\right| \psi>\left.\right|^{2} \\
=\frac{|<\uparrow| \psi>\left.\right|^{2}}{N}+|<\uparrow| \psi>\left.\right|^{4} \frac{N^{2}-N}{N^{2}} .
\end{gathered}
$$

Thus

$$
<\psi^{N}\left|\left(f_{\uparrow}^{N}-|<\uparrow| \psi>\left.\right|^{2}\right)^{2}\right| \psi^{N}>=\frac{1}{N}\left(|<\uparrow| \psi>\left.\right|^{2}-|<\uparrow| \psi>\left.\right|^{4}\right) \rightarrow 0 \text { as } N \rightarrow \infty
$$

and we have indeed shown

$$
\left\|f_{\uparrow}^{N}\left|\psi^{N}>-\right|<\uparrow|\psi>|^{2} \mid \psi^{N}>\right\| \rightarrow 0
$$

which is $(2.6)$.

The above derivation of the probability interpretation of ordinary quantum mechanics can be cast into the language used to interpret the cosmological wave function. "Superspace" is the configuration space $C^{N}$ of the ensemble. Equations (2.10) and (2.15) show that for large $N$ the wave function of an ensemble of identical systems, (2.5), is increasingly sharply peaked in the variable corresponding to a measurement of the frequency of spin $-\uparrow$ in the ensemble. The value about which it is peaked is $|\langle\uparrow \mid \psi\rangle|^{2}$. Thus, we predict from the $\Psi$ of (2.5) that a measurement of the frequency should yield this value.

This correspondance in language, however, points up an incompleteness in the interpretation of the cosmological wave function. To give a precise meaning to "sufficiently peaked" a measure is needed on configuration space. In ordinary quantum mechanics this is supplied by Hilbert space as the above derivation shows. However, there is no satisfactory Hilbert space formulation of quantum gravity. The reason is the problem of time. 


\subsection{The Problem of Time}

Time plays a central and peculiar role in the formulation of Hamiltonian quantum mechanics. The scalar product specifying the Hilbert space of states is defined at one instant of time. States specify directly the probabilities of observations carried out at one instant of time. Time is the sole observable not represented by an operator in Hilbert space but rather enters the theory as a parameter describing evolution. In the construction of a quantum theory for a specific system, the identification of the time variable is a central issue.

In non-relativistic classical physics time plays a special role which is unambiguously transferred to quantum mechanics. In special relativistic quantum mechanics there is already an issue of the choice of time variable, but there is also a resolution. We can construct quantum mechanics using as the peculiar time variable the time associated with a particular Lorentz frame. The issue is whether the quantum theory, so constructed, is consistent with the equivalence of Lorentz frames. It is. There is a unitary relation between the quantum theories constructed in different Lorentz frames and physical amplitudes are therefore Lorentz invariant.

For the construction of quantum theories of spacetime the choice of time becomes a fundamental difficulty. A preferred foliation of spacetime by spacelike surfaces is necessary to formulate canonical quantum mechanics. The classical theory certainly singles out no such foliation, and we have no evidence that theories formulated on two different foliations are unitarily equivalent. There is thus a conflict between canonical quantum mechanics and general covariance.

To resolve this conflict we have, it seems to me, two choices (1) Modify general relativity so at the quantum mechanical level a preferred time is singled out, or (2) Modify quantum mechanics so it does not need a preferred time.

The first option has been much discussed. In these lectures I would like to offer a few thoughts about the second.

Feynman's sum over histories formulation of quantum mechanics is a natural alternative starting point for constructing quantum theories of spacetime in which the problem of time is neither as immediate nor as central as it is in Hamiltonian quantum mechanics. The basic ingredients of a sum over histories formulation are these:

(1) The Histories: A history $\mathcal{H}$ is the set of observables which describe all possible experiments. Examples are the particle paths of ordinary quantum mechanics or the 4 -geometries of spacetime physics.

(2) The Probability Amplitude for a History. The joint probability amplitude for making all the observations which make up a history is

$$
\Phi(\mathcal{H})=\exp [i S(\mathcal{H})],
$$

where $S(\mathcal{H})$ is the classical action for the history. For a particle this is

$$
S[X(\tau)]=\int_{\tau^{\prime}}^{\tau^{\prime \prime}} d \tau\left[\frac{1}{2} m \dot{X}^{2}-V(X)\right],
$$


or for spacetime with the dynamics of general relativity it is

$$
S\left[{ }^{4} \mathcal{G}\right]=\frac{1}{\ell^{2}} \int_{M} d^{4} x R \sqrt{-g}-\frac{2}{\ell^{2}} \int_{\partial M} d^{3} x h^{\frac{1}{2}} K .
$$

(3) Conditional Probability Amplitudes. In particular experiments the observables can be divided into three classes (i) The conditions $\mathcal{C}$ - those observables fixed by the experimental arrangement. (ii) The observations $\mathcal{O}$ - the results of the experiments. (iii) The unobserved $\mathcal{U}$ - those observables neither conditioned nor observed. The conditional amplitude for $\mathcal{O}$ given $\mathcal{C}$ is (the principle of superposition)

$$
\Phi(\mathcal{O} \mid \mathcal{C})=\sum_{\mathcal{U}} \Phi(\mathcal{U})
$$

A measure, which is just as important as the action, is needed to define such sums.

(4) Probability. The relative probability that $\mathcal{O}$ occurs in a set of observations given the conditions $\mathcal{C}$ is $|\Phi(\mathcal{O} \mid \mathcal{C})|^{2}$. From this, the probabilities of one outcome of an exhaustive and exclusive set of observations can be computed by appropriate normalization according to the usual rules for probability.

Some further restrictions and caveats must be given, but this is the basic framework. The ideas will perhaps become clearer with an example illustrated in Figure 1. We consider a non-relativistic particle moving in one dimension. Suppose the particle starts at $X_{1}$ at time $\tau_{1}$. It then passes through a slit of width $\Delta_{2}$ at time $\tau_{2}$. At $\tau_{3}$ there is a coherent detector which registers whether the particle is in the interval $\Delta_{3}$ disturbing the particle as little as possible. Finally at $\tau_{4}$ the particle's position is detected. The conditions $\mathcal{C}$ for this experiment might be those imposed at times $\tau_{1}$ and $\tau_{2}$. A complete and exclusive set of observations would then be, $X_{4}$, the value of the position at $\tau_{4}$ and whether the detector at $\tau_{3}$ registered ("clicked") or did not. Given the experimental arrangement one of these possibilities must happen and no more than one can happen.

Let us compute the probability for the detector to click and the particle to be found at $X_{4}$ in a range $\Delta_{4}$. The conditional probability amplitude $\Phi\left(X_{4}\right.$, click $\left.\mid \mathcal{C}\right)$ is the sum over all paths which start at $X_{1}$ at $\tau_{1}$ pass through the slit at $\tau_{2}$, cross the detector volume at $\tau_{3}$ and end at $\tau_{4}$ at $X_{4}$. (Figure 1$)$. The conditional amplitude $\Phi\left(X_{4}\right.$, noclick $\left.\mid \mathcal{C}\right)$ would be a similar sum over paths which cross outside the detector volume at $\tau_{3}$. The probability is the square of $\Phi\left(X_{4}\right.$, click $\left.\mid \mathcal{C}\right)$ normalized over the set of complete and exclusive possibilities. That is

$$
\begin{aligned}
& P\left(X_{4} \text { in } \Delta_{4}, \operatorname{click} \mid \mathcal{C}\right)=\Delta_{4}\left|\Phi\left(X_{4}, \operatorname{click} \mid \mathcal{C}\right)\right|^{2} \\
& \quad \times\left[\int_{R} d X_{4}\left|\Phi\left(X_{4}, \operatorname{click} \mid \mathcal{C}\right)\right|^{2}+\int_{R} d X_{4} \mid\left.\Phi\left(X_{4}, \text { noclick } \mid \mathcal{C}\right)\right|^{2}\right]^{-1} .
\end{aligned}
$$

The same result is predicted by ordinary quantum mechanics [10]. There one would say that the state at time $\tau_{1}$ was $\left|X_{1} \tau_{1}\right\rangle$, the state with the particle localized at $X_{1}$. After the localization at $\tau_{2}$ the "wave packet is reduced" and the state is

$$
\left|\psi_{2}\right\rangle \equiv N_{2}^{-2} \int_{\Delta_{2}}\left|x_{2} \tau_{2}\right\rangle\left\langle x_{2} \tau_{1} \mid X_{1} \tau_{1}\right\rangle
$$




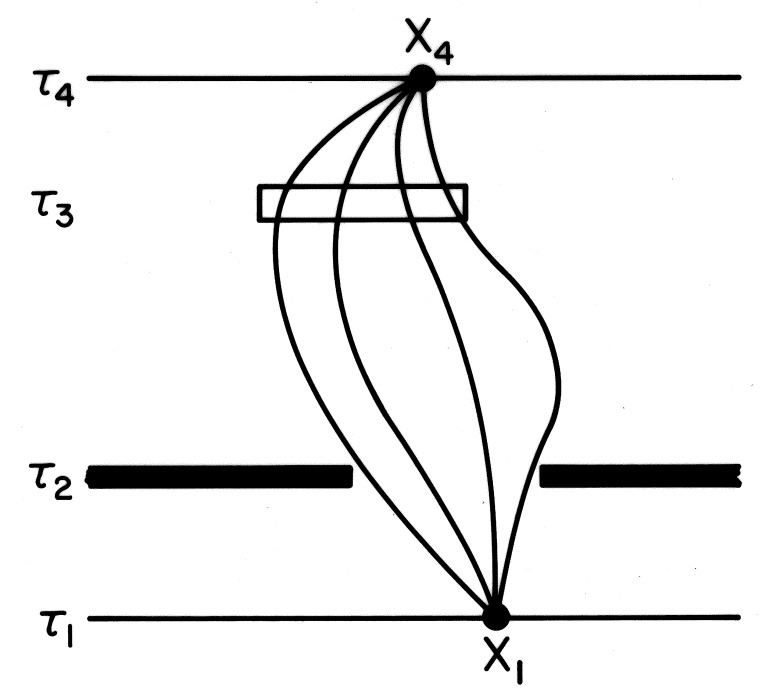

Figure 1: An example illustrating the construction of conditional probability amplitudes. A particle is localized at $\tau_{1}$, passes through a slit at $\tau_{2}$, registers or does not register in a detector volume at $\tau_{3}$, and its position is determined at $\tau_{4}$. Given that the particle started at $X_{1}$ and passed through the slit, the conditional probability amplitude for it to register in the detector and be at $X_{4}$ is a sum over all paths which start at $X_{1}$ pass through the slit and detector volume and end at $X_{4}$.

with $N_{2}$ determined so the state has unit norm. At $\tau_{3}$ the "wave packet is again reduced." The probability that the particle is inside the detector at $\tau_{3}$ is

$$
P(\text { click })=\int_{\Delta_{3}} d x_{3}\left|\left\langle x_{3} \tau_{3} \mid \psi_{2}\right\rangle\right|^{2}
$$

The state after detection is the normalized projection of $\mid \psi_{2}>$ on the interval $\Delta_{3}$

$$
\left|\psi_{3}\right\rangle=N_{3}^{-2} \int_{\Delta_{3}} d X_{3}\left|X_{3} \tau_{3}\right\rangle\left\langle X_{3} \tau_{3} \mid \psi_{2}\right\rangle .
$$

At $\tau_{4}$ the probability that the particle is at $X_{4}$ and the detector has clicked is the product

$$
\Delta_{4}\left|\left\langle X_{4} \tau_{4} \mid \psi_{3}\right\rangle\right|^{2} P(\text { click })
$$

This is the same as (2.20) as an explicit calculation will show.

The contrast between the usual discussion of this experiment and that in the sum over histories formulation shows that the sum over histories formalism handles observations at different times democratically and efficiently, so that it is well adapted to deal with conditions and observations which are not in temporal order. In particular, the sum over histories formulation can deal efficiently and naturally with observations which lie on a general surface, $\tau=\tau(X)$, and with conditions which also lie on the surface. It is thus especially useful in cosmology. 


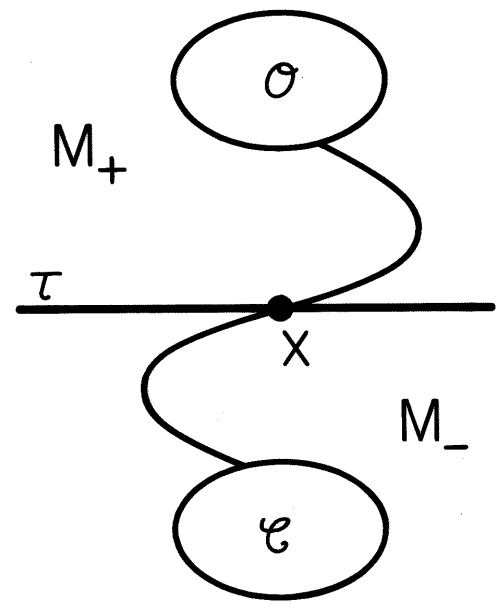

(a)

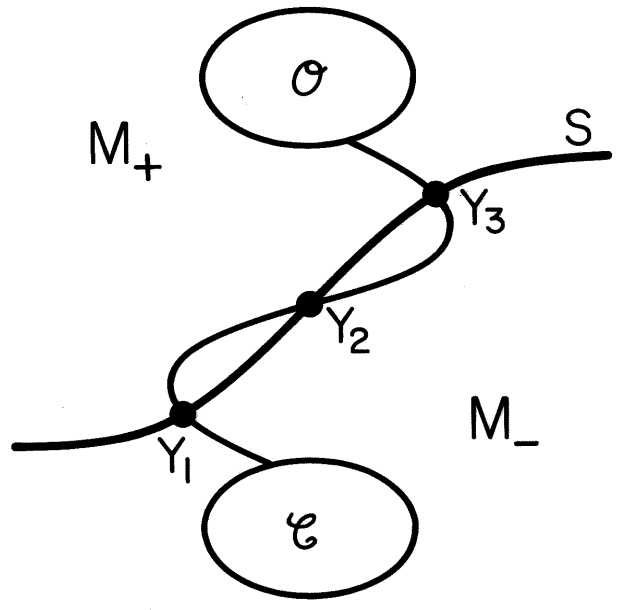

(b)

Figure 2: (a) When conditions, $\mathcal{C}$, and observations, $\mathcal{O}$, are in temporal order, a path contributing to the conditional amplitude $\Phi(\mathcal{O} \mid \mathcal{C})$ intersects an intermediate surface of constant preferred non-relativistic time $\tau$ at one and only one position $X$. The sum defining the amplitude may thus be factored in to a sum over paths prior to $\tau$, a sum over paths after $\tau$ and a sum over $X$. (b) By contrast, a path contributing to the conditional amplitude $\Phi(\mathcal{O} \mid \mathcal{C})$ may intersect a general surface $S$ many times. Indeed, the expected number of intersections is infinite.

We can now ask whether we can recover the Hilbert space formulation of quantum mechanics from its sum over histories version. This is easy to do on the surfaces of the preferred non-relativistic time. A conditional amplitude $\Phi(\mathcal{O} \mid \mathcal{C})=\sum_{\text {paths }} e^{i S}$ for which the conditions and observations are temporally ordered can be factored into a sum over paths before an intermediate surface $\tau$ and a sum after $\tau$ (Figure 2a)

$$
\Phi(\mathcal{O} \mid \mathcal{C})=\int d X \psi_{\mathcal{O}}^{*}(X \tau) \psi_{\mathcal{C}}(X \tau)
$$

where $\psi_{\mathcal{C}}(X \tau)=\sum_{\text {paths in } M_{-}} e^{i S}$ for paths which meet the conditions $\mathcal{C}$ and end at $X$. There is a similar expression for $\psi_{\mathcal{O}}^{*}$. This sum defines the wave function from the sum over paths. Further, it has its usual probability interpretation because the positions at $\tau$ are a set of exhaustive and exclusive observations.

How would this construction go on a more general surface? The crucial difference is that now the paths can cross and recross the surface many times. (Figure 2b). Formally, since each path is divided into parts by the surface, one could write down

$$
\Phi(\mathcal{O} \mid \mathcal{C})=\sum_{\substack{\text { number of } \\ \text { crossings, } n}} \int d Y_{1} \cdots d Y_{n} \psi_{\mathcal{O}}^{*}\left(Y_{1} \cdots Y_{n}, S\right) \psi_{\mathcal{C}}\left(Y_{1} \cdots Y_{n}, S\right)
$$


where

$$
\psi_{\mathcal{C}}=\sum_{\text {paths in }} e^{i S}
$$

Some attention is now needed to define what one means by sums over regions like $M_{-}$. This can be done by introducing a spacetime lattice and going to Euclidean time. Then there is a close connection between sums over histories and the continuum limits of stochastic processes. In particular, for a free particle the sums over histories can be defined as the continuum limit of a random walk.

When one calculates the sum for a fixed number of crossings in this manner, one finds that the amplitude vanishes! A composition law of the form (2.27) exists on the lattice but does not have a continuum limit. We do not recover a Hilbert space formulation of quantum mechanics on a general surface $\tau=\tau(X)$. The reason is that the expected number of crossings is infinite and the amplitude for any finite number of crossings is zero. Only due to the peculiar fact that the paths move forward in the preferred nonrelativistic time can we recover a Hilbert space formulation of the theory on surfaces of that time because we know the paths cross them once and only once.

The absence of a Hilbert space for a general surface is not an obstacle to the computation of probabilities for observations and conditions which lie on the surface. The sum over histories formulation allows this and our specific example illustrates it. However, typically, greater care is needed to pose questions with sensible answers (unlike the amplitude of (2.28)).

Now imagine for a moment that we had been brought up on sum over histories quantum mechanics. What would our attitude be if we encountered a theory in which there was no surface on which we could recover a Hamiltonian, Hilbert space formulation of the theory? Would we insist on this as a necessary requirement for a successful quantum theory? I would like to suggest that the answer should be no.

I would now like to describe two examples of theories in which it seems is not possible to recover a Hilbert space formulation, at least not by the methods we have been describing. The first is non-relativistic quantum mechanics with real clocks. The second is general relativity.

We have no direct perception of the time of non-relativistic mechanics. Psychological time is certainly poorly correlated with this variable. What we do observe are the positions of indicators in mechanical systems carefully arranged so these indicators are correlated with the time of the Schrödinger equation. Such systems are called clocks. The simplest example is a free particle moving with definite velocity in one dimension. Its position $T$, appropriately calibrated, gives the time.

In quantum mechanics an ideal clock would be one whose position $T$ remained always correlated with the Schrödinger equation time $\tau$. A possible solution to the Schrödinger equation is then

$$
\psi(T, \tau)=\delta(T-\tau)
$$

The corresponding Hamiltonian would be linear in the momenta

$$
h_{C}=-i \frac{\partial}{\partial T}=P_{T}
$$

Such clocks do not exist. The energy of this Hamiltonian would be unbounded below. 
Real clocks, such as our free particle, can exist. Their energy is positive because their Hamiltonians are quadratic in the momenta

$$
h_{C}=\frac{P_{T}^{2}}{2 M}
$$

However, such clocks are inevitably imperfect. In the case of a particle even if there is initially a sharp correlation between $T$ and $\tau$, eventually the wave packet will spread and the clock will lose accuracy. The spreading can be reduced arbitrarily by making the particle sufficiently massive. Such general limitations on the masses of clocks have been discussed by Salecker and Wigner [11]. These limitations are not important in non-relativistic quantum mechanics because one can imagine arbitrarily massive clocks which do not disturb the system. In the gravitational physics of closed systems, however, these limitations become fundamental.

Suppose that we try and construct a non-relativistic quantum mechanics in which the indicators of real clocks are involved directly. This is a problem which has been discussed by DeWitt [12], Peres [13], Page and Wooters [14] and no doubt by many others. From the sum over histories point of view one might proceed as follows: The histories are the world lines $X(\tau), T(\tau)$; moving forward in $\tau$ but both forward and backward in $T$. The observables and conditions will involve $T$ and $X$ and therefore sums over the unobserved parameter $\tau$. An interesting set of correlations are the values of $X$ for a given $T$. If a further part of the conditions is to put the $T$ - component of the system into "a good clock state" we recover an approximation to the predictions of ordinary quantum mechanics. By making the clocks massive we can make this approximation as accurate as desired. For no finite mass, however, do we recover a Hilbert space formulation of the theory on surfaces of constant $T$. Formulated in terms of $T$ and $X$ there are no preferred surfaces, and the paths cross and recross a given $T$ an arbitrarily large number of times.

My last example is general relativity and, in particular, the quantum mechanics of closed cosmologies. The histories, as will be described in more detail in the next section, are cosmological 4-geometries. There is no natural time parameter for a cosmological history. That is, there is no parameter constructed from the metric and matter fields which, for a general history, defines a foliating family of spacelike hypersurfaces such that the parameter takes a distinct value on each surface. Put differently, for a candidate parameter such as $\sqrt{h}$ or $K$, one can find histories that move forward and backward through a given value an arbitrarily large number of times. There is no preferred time. It seems unlikely, therefore, that one will recover a Hilbert space of states from sum over histories quantum mechanics by the analog of straightforward construction used for a particle earlier in this section. I would like to suggest, however, that, even in the absence of a Hilbert space formulation, one can formulate a predictive quantum mechanics of the single system which is our universe using the sum over histories formulation I have described. Correlations involving both conditions and observations on a spacelike surface can be investigated using the measure supplied by the sum over histories. Predictions of correlations verifiable for the single system can be made if the wave function is sufficiently peaked or sufficiently small. This formulation is but a slight modification of Hamiltonian quantum mechanics which coincides with that formulation when a Hamiltonian theory is available. In the following sections we shall discuss some first steps towards the 
implementation of this program.

\section{Laws for Initial Conditions}

\subsection{The Sum Over Histories Formulation of Quantum Cosmol- ogy}

To apply the sum over histories formulation of quantum mechanics to cosmology three things are needed: the histories, the action, and the measure. The histories are cosmological spacetimes with matter fields. For simplicity we shall take the spacetimes to be spatially closed, Lorentzian 4-geometries whose topology is of the form $\mathbf{R} \times M^{3}$ with $M^{3}$ a compact 3-manifold (typically the 3 -sphere). There is no compelling reason for this restriction on topology, and indeed it is interesting to investigate other possibilities [15], but this assumption will simplify our discussion without limiting the central ideas.

There is, today, no choice for the action of spacetime coupled to matter which yields a satisfactory quantum field theory judged by familiar local standards. Whatever the correct theory, we expect that its low energy limit will be Einstein gravity coupled to matter. The action for Einstein gravity on a spacetime region $M$ is

$$
\ell^{2} S_{E}[g]=\int_{M} d^{4} x(-g)^{\frac{1}{2}} R-2 \int_{\partial M} d^{3} x(h)^{\frac{1}{2}} K .
$$

Here, $R$ is the scalar curvature, $\ell=(16 \pi G)^{\frac{1}{2}}$ is the Planck length, $\partial M$ is the boundary of $M, h_{i j}$ is the metric induced on the boundary by $g_{\alpha \beta}$ and $K$ is the trace of the extrinsic curvature of the boundary. The action for a free scalar field, $\Phi$, with mass $M$ is a simple representative of the many possible matter field actions,

$$
\begin{aligned}
S_{\Phi}[g, \Phi] & =-\frac{1}{2} \int d^{4} x(-g)^{\frac{1}{2}}\left[(\nabla \Phi)^{2}+\xi R \Phi^{2}+M^{2} \Phi^{2}\right] \\
& +\xi \int_{\partial M} d^{3} x(h)^{\frac{1}{2}} K \Phi^{2} .
\end{aligned}
$$

Where concrete illustrations of the action are needed, we shall use (3.1) and (3.2).

Specification of the weights with which to carry out the sum over histories is just as important for quantum mechanics as the specification of the action. The sums over paths defining the quantum mechanics of a particle may be given a concrete meaning as the limit of sums over increasingly refined piecewise linear approximations to those paths. Weights can be assigned to each piecewise linear path defining concretely a "measure" on the space of paths. Sums over geometries may be given concrete meaning as the limit of sums over piecewise flat approximations to them using the methods of the Regge Calculus [16] and in this way a "measure" on geometries can be defined [17].

Conditional probability amplitudes are formed from $\exp (i S)$ by summing over geometries and field configurations. We have argued that representative predictions in cosmology involve observations made locally, "at one moment of time," with conditions specified, in part, at the same moment of time. Such predictions are extractable from the amplitude for observations on a spacelike surface, that is, from the wave function. 
The wave function for a spacelike surface is determined by a sum over histories restricted by conditions "in the past" of the spacelike surface. Specifically we write

$$
\Psi_{C}\left[h_{i j}(\mathbf{x}), \Phi(\mathbf{x})\right]=\int_{C} \delta g \delta \Phi \exp (i S[g, \Phi]),
$$

where the sum is over cosmological 4-geometries and field configurations which match the arguments of the wave function and satisfy the conditions $C$.

The wave function on a spacelike surface is not the only conditional probability amplitude which could be computed. It is, however, the one from which we expect to deduce most interesting cosmological predictions. We do this not by calculating probabilities, for the universe is a single system. Rather, as described in Section 2, we search the configuration space for regions where the wave function is sharply peaked. These correlations are the predictions of quantum cosmology. Given a set of conditions $C$, it is a largely open, but important question, what predictions one can expect. As we shall describe in Section 4, this problem is greatly simplified if there is a region of configuration space in which the wave function can be approximated semiclassically.

A law for initial conditions in quantum cosmology is a law for the conditions $C$. That is, a law for initial conditions is a specification of the class of geometries and field configuration which are summed over in (3.3) to yield the wave function of the universe.

\subsection{Constraints}

We are not free to specify any wave function as a theory of initial conditions. It must be representable as a sum over histories of the form (3.3) reflecting the underlying gravitational dynamics. In particular, it must satisfy certain constraints which are consequences of this dynamics. We shall now briefly review these using the example of pure Einstein gravity [7].

There are four constraints in general relativity. Three of them arise from the requirement that the wave function, $\Psi\left[h_{i j}\right]$, depend only on three geometry and not on the choice of coordinates used to describe that geometry. $\Psi$ must thus be the same on two three metrics which are connected by a diffeomorphism. Infinitesimal diffeomorphisms are generated by a vector $\xi^{k}$ according to

$$
h_{i j} \rightarrow h_{i j}+D_{(i} \xi_{j)}
$$

Thus, for infinitesimal $\xi^{k}$

$$
\Psi\left[h_{i j}+D_{(i} \xi_{j}\right]=\Psi\left[h_{i j}\right]
$$

or equivalently

$$
\left.\int_{M^{3}} d^{3} x D_{(i} \xi_{j}\right) \frac{\delta \Psi}{\delta h_{i j}(\mathbf{x})}=0 .
$$

Integrating by parts on the compact manifold $M^{3}$, and recalling that $\xi^{k}$ is arbitrary, one arrives at the three constraints

$$
D_{i}\left(\frac{\delta \Psi}{\delta h_{i j}(\mathbf{x})}\right)=0 .
$$


These are called the "momentum" constraints.

The fourth constraint of general relativity arises because general relativity is an example of a parametrized theory in which time occurs as one of the dynamical variables. To illustrate the idea we begin with a simple model [7].

Consider a non-relativistic particle whose dynamics is described by the action

$$
S[X(T)]=\int d T \ell(d X / d T, X) .
$$

Express both $X$ and $T$ as functions of a parameter $\tau$ and thereby introduce the time $T$ as a dynamical variable in the action

$$
S[X(\tau), T(\tau)]=\int d \tau \dot{T} \ell(\dot{X} / \dot{T}, X),
$$

where a dot denotes a $\tau$-derivative. This action is invariant under reparametrizations of the label time

$$
\tau=f\left(\tau^{\prime}\right), X^{\prime}\left(\tau^{\prime}\right)=X\left(f\left(\tau^{\prime}\right)\right), T^{\prime}\left(\tau^{\prime}\right)=T\left(f\left(\tau^{\prime}\right)\right) .
$$

If we calculate the Hamiltonian associated with the Lagrangian in (3.9), we find first that

$$
H=\dot{T}\left(p_{T}+h\right),
$$

where $p_{T}$ is the momentum conjugate to $T$ and $h$ is the Hamiltonian associated with the Lagrangian $\ell$. Second, we find that, identically,

$$
H=0 \text {. }
$$

The vanishing of the Hamiltonian is a characteristic feature of theories which are invariant under reparametrizations of the time.

In the quantum mechanics of this model, we construct the wave function $\psi_{C}(X, T)$ for a particular moment of time as a sum of $\exp (i S)$ over an appropriate class of paths, $X(T)$. We can carry out this sum in parametrized form - integrating over histories $X(\tau), T(\tau)$ and using the action (3.9). However, histories which differ only by a reparametrization of $\tau$ [eq. (3.10)] correspond to the same path. To count these only once in the sum over histories we can "fix" the parametrization by requiring a particular relation between $\tau$ and $T$

$$
\tau=F(T),
$$

for arbitrary increasing $F(T)$ and write the sum over histories as

$$
\psi_{C}(X, T)=\int_{C} \delta X \delta T\left|\frac{d F}{d T}\right| \delta(\tau-F(T)) \exp (i S[X, T]) .
$$

The functional $\delta$ - function is the analog of the "gauge fixing $\delta$ - function" for gauge theories and $|d F / d T|$ is the analog of the "Faddeev-Popov determinant."

The familiar sum over histories for the quantum mechanics of a non-relativistic particle is recovered from (3.14) by doing the integral over $T$ (most easily by choosing $F=T$ ). 
From this, and therefore from (3.14), the Schrödinger equation follows. Writing it in the form

$$
\left(-i \frac{\partial}{\partial T}+h\right) \psi_{C}(X, T)=0
$$

we see that it is the operator form of the constraint $H=0$. Thus the classical constraints arising from invariance under reparametrization of the time are enforced as operator relations in quantum mechanics. One sees that the vanishing of the Hamiltonian for a parametrized theory does not mean the absence of dynamics, it is the dynamical relation.

General relativity is invariant under the group of diffeomorphisms in four dimensions. There are correspondingly four constraints. They can be written in " $3+1$ form" by choosing a family of spacelike surfaces and using as basic variables their intrinsic metric, $h_{i j}$, and extrinsic curvature, $K_{i j}$.

Three of the four constraints express the invariance under diffeomorphisms in the 3-surface. These are the constraints we have already discussed. The fourth constraint expresses the invariance of the theory under choice of the choice of spacelike surfaces, that is, under reparametrizations of the time. As in the simple model, the constraint is that the total Hamiltonian (density) vanish. For this reason it is called the Hamiltonian constraint. Classically its form is

$$
H=\ell^{2} G_{i j k \ell} \pi^{i j} \pi^{k \ell}+\ell^{-2} h^{\frac{1}{2}}\left(-{ }^{3} R+2 \Lambda\right)=0
$$

where ${ }^{3} R$ is the scalar curvature of the 3 -surface, $\pi^{i j}$ are the momenta conjugate to $h_{i j}$

$$
\ell^{2} \pi_{i j}=h^{1 / 2}\left(K_{i j}-h_{i j} K\right)
$$

and $G_{i j k \ell}$ is the "supermetric"

$$
G_{i j k \ell}=\frac{1}{2} h^{-\frac{1}{2}}\left(h_{i k} h_{j \ell}+h_{i \ell} h_{j k}-h_{i j} h_{k \ell}\right)
$$

Quantum mechanically eq (3.17) becomes an operator constraint on the wave function called the Wheeler-DeWitt equation. It takes the form

$$
\left[-\ell^{2} \nabla_{x}^{2}+\ell^{-2} h^{\frac{1}{2}}\left(-{ }^{3} R+2 \Lambda\right)\right] \Psi\left[h_{i j}\right]=0,
$$

where

$$
\nabla_{x}^{2}=G_{i j k \ell} \frac{\delta^{2}}{\delta h_{i j}(\mathbf{x}) \delta h_{k \ell}(\mathbf{x})}+\left(\begin{array}{c}
\text { linear derivative terms } \\
\text { depending on factor ordering }
\end{array}\right) .
$$

The Wheeler-DeWitt equation follows formally from the sum over histories for quantum cosmology in much the same way that the Schrödinger equation follows from the sum over parametrized paths (3.14) (Problem 2). It may be thought of as a functional differential equation which expresses the dynamics of quantum cosmology in much the same way that the Schrödinger equation expresses the dynamics of particle quantum mechanics. 


\subsection{A Proposal for a Wave Function of the Universe}

There have been a number of proposals for a quantum state of the universe [1-5]. Perhaps the most developed of these is the proposal of Stephen Hawking and his coworkers that the wave function of the universe is determined by a sum over compact Euclidean 4 geometries. Detailed expositions of this idea can be found elsewhere [18, 6]. Here we shall just state the proposal so that there is at least one concrete idea with which to illustrate the subsequent discussion.

Euclidean sums over histories as well as Lorentzian ones may be used to construct solutions to constraints. Consider, for example, the sum over particle paths

$$
\psi_{0}\left(X_{0}\right)=\int_{C_{0}} \delta X \exp (-I[X(T)]),
$$

where $I$ is the Euclidean action for a non-relativistic particle in a potential $V(X)$

$$
I[X(T)]=\int d T\left[\frac{1}{2} M\left(\frac{d X}{d T}\right)^{2}+V(X)\right],
$$

and the sum is over all paths which start at $X_{0}$ at Euclidean time $T=0$ and proceed to a configuration of minimum action at large negative times. The wave function $\psi_{0}\left(X_{0}\right)$ so defined satisfies the constraint (3.15). It is, in fact, the ground state wave function.

Euclidean sums over 4 - geometries give solutions to the operator constraints of gravitational theories. Consider a cosmological manifold of the form $\mathbf{R}^{+} \times M^{3}$, where $\mathbf{R}^{+}$is half the real line. The manifold thus has an $M^{3}$ boundary. A sum over Euclidean 4-geometries and field configurations of the form

$$
\Psi\left[h_{i j}(\mathbf{x}), \Phi(\mathbf{x})\right]=\int_{C} \delta g \delta \Phi \exp (-I[g, \Phi]),
$$

where $I$ is the Euclidean action for Einstein gravity coupled to matter, will formally satisfy the constraints (3.7) and (3.9) provided the metric and matter field induced on the boundary by each contributor to the sum match those in the argument of the wave function [28]. (Problem 2). A particular wave function is singled out by summing over compact 4 - geometries with no other boundary and over matter field configurations which are regular on these geometries. The proposal of Hawking and his coworkers is that this is the wave function of our universe.

The Euclidean action for Einstein gravity

$$
\ell^{2} I[g]=-\int_{M} d^{4} x g^{1 / 2} R-2 \int_{\partial M} d^{3} x h^{1 / 2} K
$$

is not positive definite. Thus, for general relativity and other theories with this property the contour of integration in (3.23) cannot be over purely real metrics - the integral would diverge. The contour of integration must be taken in complex directions [19]. From the Hamiltonian perspective one is free to make this distortion as long as the correct sum over the true physical degrees of freedom is preserved. This seems to be 
possible for linearized gravity and for the sum over histories defining the ground state of isolated systems [19]. We shall presume that the analogous contour exists for closed cosmologies although this has yet to be demonstrated (Problem 3). The complex nature of the contour in the proposal (3.23) is not an inessential technicality. Were the contour purely real, the wave function would be positive and never oscillate. With a complex contour we expect oscillation in some regions of configuration space, and, as we shall see in Section 4, only in such regions does the wave function predict the correlations of classical physics.

\section{The Limit of Classical Geometry and Quantum Field Theory in Curved Spacetime}

In the context of quantum mechanics the predictions of classical physics are predictions of special kinds of correlations between special classes of observables. For example, if we measure the position and momentum of a particle at one time with accuracies consistent with the uncertainty principle and then again at a later time the laws of classical physics predict a definite correlation between these two measurements.

In Section 2 we saw how a wave function predicts correlations among the observables of an individual system. It is a very special situation when the predicted correlations of some observables are classical, but also a very important situation. There are three reasons: First, fundamentally we interpret the world in classical terms. Second, certainly in cosmology our crude observations are of classical observables. Third, as we shall describe below, it is possible to give a simple criterion - the validity of the semiclassical approximation - for when a wave function predicts classical correlations. This feature greatly simplifies extracting predictions in quantum cosmology.

The discussion of this section is an attempt at one synthesis of ideas which have had a long history in general relativity. Some notable contributions have been those of Salecker and Wigner [11], DeWitt [12], Wheeler [25], Peres [13], Page and Wooters [14], Banks [21], Hawking and Halliwell [22], D'Eath and Halliwell [23], and Brout, Horwitz and Weil [24].

\subsection{The Semiclassical Approximation to Non-Relativistic Par- ticle Quantum Mechanics}

Let us recall the semiclassical approximation to the wave function of a state of definite

energy in non-relativistic particle quantum mechanics. Assuming that the Hamiltonian has been normalized so that the energy is zero, we want to solve

$$
H \psi(X)=0
$$

where

$$
H=-\frac{\hbar^{2}}{2 M} \frac{d^{2}}{d X^{2}}+V(X)
$$


To obtain the semiclassical approximation we write

$$
\psi(X)=\exp [i S(X) / \hbar],
$$

and expand $S(X)$ in powers of $\hbar$

$$
S=S_{0}+\hbar S_{1}+\cdots
$$

Writing out the Schrödinger equation to the lowest order, $\hbar^{0}$, one finds

$$
\frac{1}{2 M}\left(\frac{d S_{0}}{d X}\right)^{2}+V(X)=0,
$$

so that $S_{0}$ obeys the classical Hamilton Jacobi equation and, indeed, is given by $\pm \int^{x} \sqrt{-2 m V(X)} d X$. In regions where $V(X)<0$ (the "classically allowed" regions for $E=0)$ there is a real solution for $S_{0}$ and the wave function oscillates. In the classically forbidden regions $S_{0}$ must be complex, $S_{0}=i I_{0}$, where $I_{0}$ solves the "Euclidean" Hamiltonian Jacobi equation. The wave function in these regions is a sum of real exponentials.

The next order is also easy to compute. The order $\hbar$ part of the Schrödinger equation is

$$
i \frac{d^{2} S_{0}}{d X^{2}}-2 \frac{d S_{0}}{d X} \frac{d S_{1}}{d X}=0,
$$

which is easily solved for $S_{1}$. The result, for example in the classically allowed region is

$$
\psi=\left(\frac{d S_{0}}{d X}\right)^{-\frac{1}{2}} \exp \left(i S_{0} / \hbar\right) .
$$

In the classically forbidden region the order $\hbar^{0}$ approximation is modified by a prefactor in the same way. Approximate solutions satisfying given boundary conditions are built by taking linear combinations of (4.7) and its complex conjugate and of the two possible exponential behaviors and matching them across the boundaries between the classically allowed and classically forbidden regions.

In a classically allowed region the interpretation of the semiclassical approximation is straightforward. Suppose measurements of the particle's position and momentum are made with accuracies consistent with the uncertainty principle. For position measurements which yield the value $X_{0}$ the wave function (4.7) is sharply peaked about the momentum

$$
p\left(X_{0}\right)=\left(\frac{d S_{0}}{d X}\right)_{X=X_{0}} .
$$

This is because near $X_{0}$

$$
S_{0}(X) \approx S_{0}\left(X_{0}\right)+\left(\frac{d S_{0}}{d X}\right)_{X=X_{0}}\left(X-X_{0}\right)+\cdots
$$

with the higher terms being negligible because $S_{0}$ is slowly varying. In this approximation, (4.7) is a wave function of definite momentum (4.8). Thus, a semiclassical 
approximation of the form (4.7) predicts this classical correlation between position and momentum. In particular, if successive measurements are made which do not disburb either position or momentum by a large amount, values must be found which are consistent with the classical equations of motion

$$
M \frac{d X}{d \tau}=p(X)=[-2 M V(X)]^{\frac{1}{2}} .
$$

In this way classical physics is recovered.

One should stress that classical physics is recovered only in the sense of certain correlations and that the nature of these correlations depends on the form of the semiclassical approximation. For example, the wave function (4.7) does not predict much about the position of the particle but only the correlation (4.8) between position and momentum, and that implied by (4.10) between present position and future position. Of course, there are wave packet states in which both position and momentum would be predicted, but these do not have definite energy. A semiclassical wave function of the form

$$
\psi(X)=\left(\frac{d S_{0}}{d X}\right)^{-\frac{1}{2}} \cos \left[S_{0}(X) / \hbar\right]
$$

would not even predict a correlation of position with momentum but only with its absolute value. That is, it would predict that three successive measurements of position would be correlated according to the equation of motion (4.10) with the sign of $p(X)$ determined from the first two.

\subsection{The Born-Oppenheimer Approximation for Real Clocks}

In non-relativistic quantum mechanics time is an external parameter labeling different measurements. It is not itself an observable. (See, for example, the preceding discussion of correlations in the semiclassical approximation.) We have access to this time only through the observation of correlations between the positions of clock indicators and the variables of the system. It should, therefore, be sufficient for prediction to formulate quantum mechanics entirely in terms of these variables. In the case of the quantum mechanics of a particle we would write

$$
\psi=\psi(T, X)
$$

where $X$ is the particle's position and $T$ the position of a clock indicator. We could then study $\psi$ for the correlations between $T$ and $X$ or between $T$ and other observations.

The wave function in such a formulation of quantum mechanics will satisfy a constraint reflecting invariance under the choice of parameter used to label the histories of $T$ and $X$. Indeed, the parametrized time non-relativistic quantum mechanics of Section 3.3 is a model for this kind of theory. The constraint (3.15) of that theory was

$$
H \psi=\left(-i \frac{\partial}{\partial T}+h\right) \psi=0
$$


If we read this as the requirement that the total Hamiltonian vanish, the variable $T$ may be thought of as the position of a kind of ideal clock whose Hamiltonian is

$$
h_{C}=-i \frac{\partial}{\partial T}=P_{T}
$$

The Hamiltonian (4.14) is a rather poor model of a real clock. Among other unrealistic features, its spectrum is unbounded below. The Hamiltonians of more realistic clocks we expect to be quadratic in their momenta. A particle moving freely in one dimension with narrow dispersions in position and momentum is a simple example. The position of the particle is a measure of time. In such a theory, as we shall show below, we recover the classical notion of time only in the approximation in which the dynamics of the clock can be treated semiclassically.

The constraints of general relativity are also quadratic in the momenta. In the case of closed cosmologies it does not even seem possible to approximate the notion of an ideal clock [25]. Here too we shall recover a notion of time in the quantum theory only in the approximation in which spacetime is treated semiclassically. We shall discuss spacetime in the next subsection. Here, we begin with a simple model of a real clock due to Banks $[21,24]$ which illustrates the central features of these ideas.

Consider a system consisting of a particle described by a position $X$ and a clock with an indicator variable $T$. We consider a constraint of the form

$$
H \Psi=\left(h_{C}+h\right) \psi=0
$$

where $h$ is the Hamiltonian of the particle and $h_{C}$ the Hamiltonian of the clock. The dynamics of the clock we take to be specified by the action

$$
S[T(\tau)]=M \int d \tau\left(\frac{1}{2} \dot{T}^{2}+V_{C}(T)\right)
$$

so that $h_{C}$ will be quadratic in the momenta. The quantity $M$ is the mass of the clock although it also controls the coupling to the potential $V_{C}$.

In the limit $M \rightarrow \infty$ the clock motion can be treated classically. Physically, this is because, for given energy, as the mass becomes large the quantum fluctuations become small. Mathematically, it is because the classical limit in a sum over histories occurs as $\hbar \rightarrow 0$ in $\exp (i S / \hbar)$ leading to destructive interference in the sum for all but the classical trajectory. The limit $M \rightarrow \infty$ is the same limit but for the clock part of the action alone. The approximation of large $M$ is therefore not strictly the semiclassical approximation. Rather it is the analog of the Born-Oppenheimer approximation in molecular physics in which the motion of the massive nuclei are considered classically while the electronic cloud is treated quantum mechanically.

In the large $M$ limit we look for a solution of the constraint equation (4.15) of the form

$$
\psi=e^{i S(T)} \chi(T, X)
$$

where

$$
S=M S_{0}+S_{1}+M^{-1} S_{2}+\cdots
$$




$$
\chi=\chi_{0}+M^{-1} \chi_{1}+\cdots
$$

Since

$$
h_{C}=-\frac{1}{2 M} \frac{d^{2}}{d T^{2}}+M V_{C}(T)
$$

we have, on writing out the constraint (4.15),

$$
\left\{-\frac{1}{2 M}\left[i \frac{d^{2} S}{d T^{2}}-\left(\frac{d S}{d T}\right)^{2}\right]+M V_{C}\right\} \chi-\frac{1}{2 M} \frac{\partial^{2} \chi}{\partial T^{2}}-\frac{i}{M} \frac{d S}{d T} \frac{\partial \chi}{\partial T}+h \chi=0
$$

We now insert the expansions (4.18) in (4.20) and systematically expand in powers of $M$. For the leading order we find

$$
\frac{1}{2}\left(\frac{d S_{0}}{d T}\right)^{2}+V_{C}(T)=0
$$

This is the clock's classical Hamilton-Jacobi equation. Defining $p_{T}=M\left(d S_{0} / d T\right)$ it can be written in the familiar form

$$
\frac{1}{2 M} p_{T}^{2}+M V_{C}(T)=0
$$

In the next order, $M^{0}$, one finds

$$
-\frac{1}{2}\left(i \frac{d S_{0}}{d T^{2}}-2 \frac{d S_{0}}{d T} \frac{d S_{1}}{d T}\right) \chi_{0}-i \frac{d S_{0}}{d T} \frac{\partial \chi_{0}}{\partial T}+h \chi_{0}=0
$$

This is one equation for two unknowns. To fix the remaining freedom we rewrite (4.22) by defining a classical time from the solution to the classical Hamilton-Jacobi equation (4.21). We write

$$
M \frac{d T_{0}}{d \tau}=p_{T}=M \frac{d S_{0}}{d T}
$$

Integration of this relation defines $T_{0}(\tau)$ up to an initial condition and hence $\tau$ as a function of $T$. Equation (4.20) can now be rewritten to read

$$
-\frac{1}{2}\left(i \frac{d^{2} S_{0}}{d T^{2}}-2 \frac{d S_{1}}{d \tau}\right) \chi_{0}-i \frac{\partial \chi_{0}}{\partial \tau}+h \chi_{0}=0
$$

where $\chi_{0}=\chi_{0}\left(T_{0}(\tau), X\right) \equiv \chi_{0}(\tau, X)$. An inner product can be defined by integrating over $X$ at constant $\tau$

$$
(\chi, \phi)=\int d X \chi^{*}(\tau, X) \phi(\tau, X)
$$

and in it we can take the expectation value of (4.24). The result is

$$
-\frac{1}{2}\left(i \frac{d^{2} S_{0}}{d T^{2}}-2 \frac{d S_{1}}{d \tau}\right)\left(\chi_{0}, \chi_{0}\right)-i\left(\chi_{0}, \frac{\partial \chi_{0}}{\partial \tau}\right)+\left(\chi_{0}, h \chi_{0}\right)=0
$$


The imaginary part of this equation is, assuming that $h$ is Hermitian,

$$
\frac{1}{2}\left(-\frac{d S_{0}}{d T^{2}}+2 \frac{d \operatorname{Im} S_{1}}{d \tau}\right)\left(\chi_{0}, \chi_{0}\right)-\frac{1}{2} \frac{d}{d \tau}\left(\chi_{0}, \chi_{0}\right)=0
$$

The real part is

$$
\frac{d\left(R e S_{1}\right)}{d \tau}\left(\chi_{0}, \chi_{0}\right)-\frac{i}{2}\left[\left(\chi_{0}, \frac{\partial \chi_{0}}{\partial \tau}\right)-\left(\frac{\partial \chi_{0}}{\partial \tau}, \chi_{0}\right)\right]+\left(\chi_{0}, h \chi_{0}\right)=0
$$

We recover a sensible quantum mechanics if we impose the condition that the inner product is conserved

$$
\frac{d}{d \tau}\left(\chi_{0}, \chi_{0}\right)=0
$$

so that from $(4.27)$

$$
-\frac{d S_{0}}{d T^{2}}+2 \frac{d S_{0}}{d T} \frac{d I m S_{1}}{d T}=0
$$

This is the usual next -after-leading-order equation for the semiclassical approximation to the $T$ motion. Equation (4.26) becomes, assuming $\chi_{0}$ normalized,

$$
\frac{d\left(R e S_{1}\right)}{d \tau}+\left(\chi_{0}, h \chi_{0}\right)=0
$$

When (4.30) and (4.31) are substituted back into the original equation (4.22) we find

$$
i \frac{\partial \chi_{0}}{\partial \tau}=\left[h-\left(\chi_{0}, h \chi_{0}\right)\right] \chi_{0}
$$

This is the Schrödinger equation for the particle moving in the "background" time $\tau$ to the extent $\left(\chi_{0}, h \chi_{0}\right)$ is constant or negligible.

The combined equation for $\operatorname{Re} S$ accurate to first order in $M$ is from (4.31) and (4.21)

$$
\frac{1}{2}\left(\frac{d R e S}{d T}\right)^{2}+M V_{C}(T)+\left(\chi_{0}, h \chi_{0}\right)=0 .
$$

This is the classical Hamilton-Jacobi equation but with a small quantum correction to the energy. It is the semiclassical back reaction equation.

\subsection{The Approximation of Quantum Field Theory in Curved Spacetime.}

The structure of the Hamiltonian constraint for general relativity - the Wheeler-DeWitt equation - is similar both in structure and origin to the constraint of the simple model just discussed. Including the energy of a scalar matter field, the Wheeler-DeWitt equation reads

$$
\left[-\frac{1}{2} \ell^{2} \nabla_{x}^{2}+\frac{1}{2} \ell^{-2} h^{\frac{1}{2}}\left(2 \Lambda-{ }^{3} R\right)+h^{\frac{1}{2}} T_{n n}\left(\Phi,-i \frac{\delta}{\delta \Phi}\right)\right] \Psi\left[h_{i j}, \Phi\right]=0 .
$$


Here $T_{n n}(\Phi, \Pi)$ is the stress-energy of the matter field expressed in terms of the field's value and momentum and projected onto the normals of the spacelike hypersurface. It is the Hamiltonian density for the scalar field. The inverse squared Planck length enters the constraint in exactly the same way as the mass of the clock did in the model problem [cf. (4.15),(4.19)]. We may therefore consider the limit when $\ell \rightarrow 0$ and expect to treat geometry semiclassically [21-24]. This is the limit when relevant length scales are large compared to the Planck length and when relevant energies are small compared to the Planck mass.

The model of Section 4.2 reveals the central features of the $\ell \rightarrow 0$ limit so clearly that we shall just sketch the parallel steps here. We write

$$
\Psi\left[h_{i j}, \Phi\right]=e^{i S\left[h_{i j}\right]} \chi\left[h_{i j}, \Phi\right]
$$

and systematically expand $S, \chi$ and the Wheeler-DeWitt equation in powers of $\ell$. In the leading order we recover the Hamilton-Jacobi equation of general relativity for $S_{0}$. From its solution we can introduce the momenta

$$
\ell^{2} \pi^{i j}(\mathbf{x})=\frac{\delta S_{0}\left[h_{i j}(\mathbf{x})\right]}{\delta h_{i j}(\mathbf{x})}
$$

The $\pi_{i j}$ are the tangent vectors to a set of integral curves in superspace which are solutions to the classical Einstein equation. For example, if we work in a gauge in which 4-metrics have the form

$$
d s^{2}=-d \tau^{2}+h_{i j}(\tau, \mathbf{x}) d x^{i} d x^{j}
$$

then classically [cf. (3.17)]

$$
\pi_{i j}=\frac{1}{2 \ell^{2} h^{\frac{1}{2}}} \frac{d}{d \tau}\left(h h_{i j}\right)
$$

Integrating

$$
\frac{d h_{i j}}{d \tau}=G_{i j k \ell} \frac{\delta S_{0}}{\delta h_{k \ell}}
$$

we recover a time dependent 4-geometry in the gauge (4.37) which satisfies the Einstein equation.

The values of $\chi_{0}$ along an integral curve in superspace define $\chi_{0}$ as a function of $\tau$.

$$
\chi_{0}=\chi_{0}\left[h_{i j}(\tau, \mathbf{x}), \Phi(\mathbf{x})\right]=\chi_{0}[\tau, \Phi(\mathbf{x})] .
$$

Then by exactly the same steps as led from (4.17) to (4.32) one finds starting from (4.35) that one ends at

$$
i \frac{\partial \chi_{0}}{\partial \tau}=\left[h^{\frac{1}{2}} T_{n n}\left(\Phi,-i \frac{\delta}{\delta \Phi}\right)-\left(\chi_{0}, h^{\frac{1}{2}} T_{n n} \chi_{0}\right)\right] \chi_{0} .
$$

This is a Schrödinger equation defining a quantum field theory in the curved background spacetime specified by a solution to (4.39). $T_{n n}$ is the Hamiltonian density for the matter field which depends on the background metric in the usual way. The inner product with 
which this equation is derived is the standard inner product in the field representation on the spacelike surface with normal $\partial / \partial \tau$ in the background (4.37), i.e.

$$
\left(\chi, \chi^{\prime}\right)=\int \delta \Phi \chi^{*}[\tau, \Phi(\mathbf{x})] \chi^{\prime}[\tau, \Phi(\mathbf{x})]
$$

Accurate to order $\ell^{2}$, the operator constraints of general relativity imply the classical constraint equations corrected by the quantum expectation value of the stress-energy of the matter field. The derivation is parallel to that of (4.33). In the Hamilton-Jacobi form in which they naturally emerge from (4.32) and (4.33) one has

$$
\begin{gathered}
\frac{1}{2}\left[-\ell^{4} G_{i j k \ell} \frac{\delta S}{\delta h_{i j}} \frac{\delta S}{\delta h_{k \ell}}+h^{\frac{1}{2}}\left({ }^{3} R-2 \Lambda\right)\right]=\frac{\ell^{2}}{2} h^{\frac{1}{2}}\left(\chi_{0}, T_{n n} \chi_{0}\right), \\
D_{j}\left(\frac{\delta S}{\delta h_{i j}}\right)=\frac{\ell^{2}}{2} h^{\frac{1}{2}}\left(\chi_{0}, T_{n}^{i} \chi_{0}\right),
\end{gathered}
$$

or equivalently using eqs. (4.36) - (4.39)

$$
\left(R_{n}^{\alpha}-\frac{1}{2} \delta_{n}^{\alpha} R\right)=\frac{\ell^{2}}{2}\left(\chi_{0}, T_{n}^{\alpha} \chi_{0}\right)
$$

where $R_{\alpha \beta}$ and $T_{\alpha \beta}$ are the Ricci curvature and stress energy expressed in an orthonormal basis one member of which is $n^{\alpha}$. These are the four constraint equations of the classical theory including the quantum corrections to the stress energy of the matter field.

The correspondence in form between (4.43) and (4.44) shows that any solution of the former will satisfy four of the quantum corrected Einstein equations. However, these Hamilton-Jacobi equations determine a solution of the full set of Einstein equations through (4.36) - (4.39). The reason is covariance. There was no intrinsic definition of the spacelike surface with normal $n^{\alpha}$. The four constraint equations (4.44) must be satisfied on any spacelike surface in the geometry. This requirement is equivalent to the full set of Einstein equations [26]. Thus,

$$
R_{\alpha \beta}-\frac{1}{2} g_{\alpha \beta} R=\frac{\ell^{2}}{2}\left(\chi_{0}, T_{\alpha \beta} \chi_{0}\right) .
$$

These are the quantum corrected ("backreaction") Einstein equations for the background geometry. (Problem 4).

The problem of extracting the predicted correlations from the wave function of the universe is in general a difficult one. As the results of this section argue, however, if the wave is well approximated by the form (4.35), with $\chi$ and $S$ given by the first few terms in an expansion in powers of the Planck length, then the correlations of a classical 4geometry containing quantum matter fields are predicted. The 4-geometry and quantum fields are defined precisely through equations $(4.36-4.39)$ and $(4.40-4.41)$. In the context of the discussion of Section 2 we envision this means the following: Imagine filling space with a system of rods, clocks and field meters which could define what is meant by measurements of distance and time to classical accuracies and measurements of 
field. The clocks, rods and meters are to be described by matter and gravitational fields so that it is possible to identify the regions in superspace consistent with various possible sets of values they might read. From the analog with non-relativistic quantum mechanics, we expect that, where the semiclassical approximation is valid, the wave function will be sharply peaked about a region consistent with values which define spacetimes satisfying the Einstein equations (4.45) and quantum fields satisfying (4.41).

In quantum cosmology, an important test of any theory of initial conditions is that there be a region of configuration space in which the wave function is well approximated semiclassically for we observe the present universe to behave classically. However, the wave function in the semiclassical approximation does not predict a unique classical history. Rather, it predicts a family of them. For example, in particle quantum mechanics a semiclassical wave function of the form $\exp [i S(X) / \hbar]$ corresponds to classical trajectories with momentum $p=d S / d X$. Information about present position must be used to single out a unique classical history. A semiclassical wave function of the form $\cos [S(X) / \hbar]$ requires even more information for it corresponds to classical trajectories with $p= \pm d S / d X$. How much present information must be used to gain definite, classical predictions from the wave function of the universe is one of the subject's most important questions.

\subsection{The Semiclassical Vacuum}

The derivation of quantum field theory in curved spacetime presented in the preceding subsection clarifies a number of issues usually regarded as internal to that subject. For example, the derivation sheds light on the meaning of the metric in the semiclassical field equation (4.45). It emerges there not as the expectation value of a field operator in some general quantum state. Rather, $g_{\alpha \beta}$ is the metric that would be determined from classical measurements of limited occuracy in a regime of configuration space in which the semiclassical approximation to a particular wave function is valid.

As a second example, the derivation of quantum field theory in curved spacetime connects the choice of "vacuum" for the matter with a theory of initial conditions i.e. with a prescription for the wave function of the universe. If the semiclassical approximation is valid, $\chi_{0}[\tau, \Phi(\mathbf{x})]$ is determined by $\Psi$ through (4.35), (4.40) and this functional defines a quantum state of the matter field in the classical background spacetime in the Hilbert space defined by (4.42). We shall call it the quantum state of the matter fields.

The determination of the quantum state of the matter fields by a prescription for the wave function of the universe may be instructively illustrated in a simple minisuperspace model. For the prescription we consider that of Section 3.3. For the minisuperspace model we restrict attention to homogeneous and isotropic geometries containing a single conformally invariant scalar field. This model is easy to analyse even it it is not very realistic.

The metric of a homogeneous isotropic geometry can be put in the form

$$
\begin{gathered}
d s^{2}=\sigma^{2}\left[-d \tau^{2}+a^{2}(\tau) d \Omega_{3}^{2}\right] \\
=\sigma^{2} a^{2}(\eta)\left[-d \eta^{2}+d \Omega_{3}^{2}\right]
\end{gathered}
$$


where $\sigma^{2}=\ell^{2} / 24 \pi^{2}$ is a convenient normalizing constant. The geometry of a three surface of constant $\tau$ is then characterized solely by its radius $a_{0}$. The wave function is a function of $a_{0}$ and a functional of the matter field on this surface.

$$
\Psi=\Psi\left[a_{0}, \Phi_{0}(\mathbf{x})\right] .
$$

Equivalently, we could expand any field configuration in harmonics

$$
\Phi(\eta, \mathbf{x})=\sum_{n} \Phi^{(n)}(\eta) Y_{(n)}(\mathbf{x})
$$

where the $Y_{(n)}(\mathbf{x})$ are standard harmonics on the 3-sphere. Then

$$
\Psi=\Psi\left[a_{0}, \Phi_{0}^{(1)}, \Phi_{0}^{(2)}, \Phi_{0}^{(3)}, \cdots\right] .
$$

The action is the sum of the Euclidean gravitational action (3.24) and the Euclidean action for the scalar field corresponding to (3.2) with $\xi=1 / 6$. The Euclidean gravitational action restricted to the minisuperspace geometries is

$$
I_{E}=\frac{1}{2} \int d \eta\left[-\left(a^{\prime}\right)^{2}-a^{2}+H^{2} a^{4}\right]
$$

where a prime denotes an $\eta$-derivative and $H^{2}=\Lambda / 3$. The matter action is considerably simplified by a dimensional rescaling

$$
\Phi(x)=\varphi(x) /\left(2 \pi^{2} \sigma^{2}\right)^{\frac{1}{2}}
$$

and by a conformal rescaling

$$
\varphi^{(n)}(\eta)=\chi^{(n)}(\eta) / a(\eta)
$$

The physics of the field, being conformally invariant, is essentially, the same in all conformally related spacetimes. The geometry is conformal to an Einstein static universe by a conformal factor $a(\eta)$. In that geometry, because of its timelike killing field, the analysis of the scalar field is considerably simplified. This is immediately apparent in the form of the matter action takes in terms of the variables $\chi^{(n)}$

$$
I_{M}=\frac{1}{2} \int d \eta\left[\left(\chi^{(n)^{\prime}}\right)^{2}+\omega_{n}^{2}\left(\chi^{(n)}\right)^{2}\right],
$$

where $\omega_{n}^{2}=\gamma_{n}+1$ and $\gamma_{n}$ are the eigenvalues of the Laplacian on the 3 -sphere.

In the minisuperspace model the wave function is given as

$$
\Psi\left[a_{0}, \chi_{0}^{(n)}\right]=\int \delta a \prod_{n} \delta \chi^{(n)} e^{-\left(I_{E}+I_{M}\right)} .
$$

The integral is over all $a(\eta)$ which correspond to compact geometries with boundary three sphere radius $a_{0}$, and over all matter mode configurations $\chi^{(n)}(\eta)$ which match 


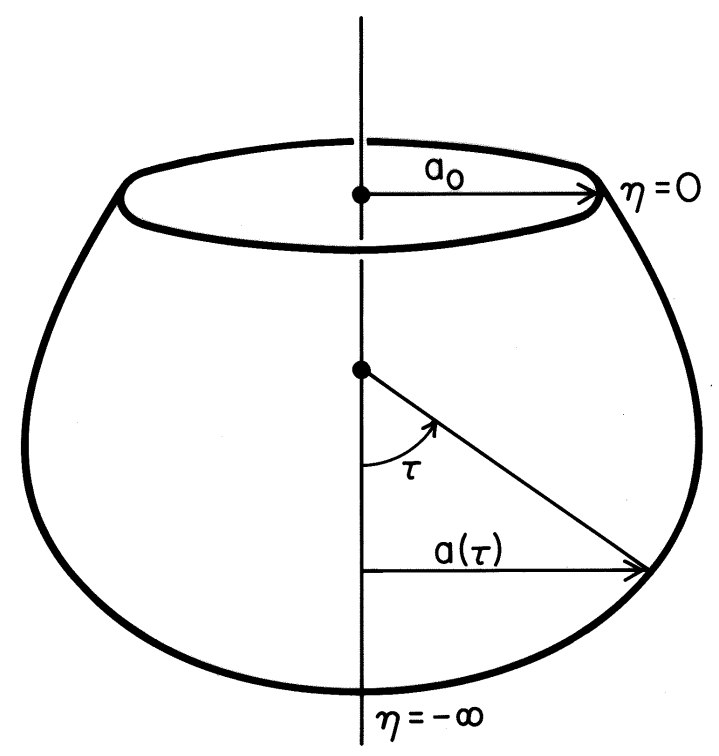

Figure 3: A two dimensional representation of a homogeneous and isotropic 4-geometry contributing to the sum for the state of minimum excitation $\Psi\left(a_{0}, \Phi_{0}\right)$. Shown embedded in a flat 3-dimensional space is a 2-dimensional slice of such a geometry whose intrinsic geometry is $\tau$ is thus a "polar angle" and $a$ the "radius from the axis." The geometry is compact and has only one boundary at which the radius is $a_{0}$, the argument of $\Psi$. The field configurations $\Phi(\tau, \mathbf{x})$ which contribute to the sum are those which are regular on this surface and which match the argument of the wave function $\Psi$ on the boundary.

$\chi_{0}^{(n)}$ on the boundary and are elsewhere regular. (Figure 3). A compact geometry will have one radius (the "south pole") at which $a$ vanishes linearly in the polar angle $\tau$. Since $d \eta=d \tau / a(\eta)$, the coordinate $\eta$ becomes logarithmically infinite at this point. We may use the last remaining gauge freedom to choose the boundary to be at $\eta=0$. The relevant coördinate range for $\eta$ is then $(-\infty, 0)$. We thus integrate over $a(\eta), \chi(\eta)$ which vanish at $\eta=-\infty$ and assume the prescribed values on the boundary.

In the simplicity of conformal invariance, the action separates into a sum of metric part and field part so that the two integrals can be done separately. The integral over the field part is trivial. One finds

$$
\Psi\left[a_{0}, \chi_{0}^{(n)}\right]=\psi\left(a_{0}\right) \prod_{n} \exp \left[-\frac{1}{2} \omega_{n}\left(\chi_{0}^{(n)}\right)^{2}\right]
$$

where

$$
\psi\left(a_{0}\right)=\int \delta a e^{-I_{E}[a]}
$$

Let us now approximate the integral for $\psi\left(a_{0}\right)$ by the method of steepest descents. For this we must find the extrema of $I_{E}$ through which the contour of integration can be distorted. We begin with values of $a_{0}$ less than $H^{-1}$. The possible extrema of $I_{E}$ are just the solutions of

$$
a^{\prime \prime}-a+2 H^{2} a^{3}=0 .
$$




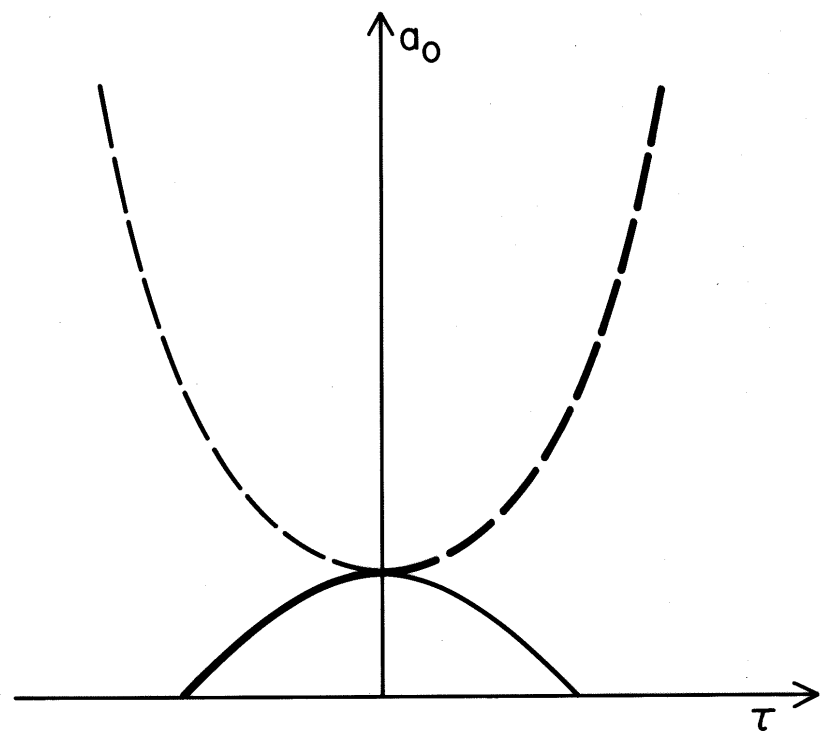

Figure 4: The extremizing scale factor for the homogeneous, isotropic minisuperspace model with conformally invariant scalar field. The solid line is the solution of (4.58) for real Euclidean extrema of the action. The complete range of $a$ from zero to maximum and back again describes the geometry of the 4-sphere (Figure 5). The dashed curve is the solution of (4.60) for complex Euclidean (Lorentzian) extrema. It describes the geometry of de Sitter space. For each value of $a_{0}$ there are thus two possible extremizing solutions. Choosing the trajectory to start on the left at $a_{0}=0$, the Euclidean prescription for the ground state singles out the heavy curve shown. This gives the semiclassical approximation to the wave function $\Psi$.

The equation has an "energy integral" whose value may be found from the regular vanishing of the $a$ at $\eta=-\infty$. Expressing this integral in terms of $\tau$ gives

$$
\left(\frac{\dot{a}}{a}\right)^{2}=\frac{1}{a^{2}}-H^{2}
$$

This is the Euclidean Einstein equation for a metric with the symmetries of the model as it must be. The solution is illustrated in Figures 4 and 5 and is just the 4 -sphere of radius $1 / H$. For $a_{0}<1 / H$ there are thus two possible extrema which are compact 4-geometries with a 3-sphere boundary of radius $a_{0}$. One for which the boundary bounds less than a hemisphere of the 4-sphere and another for which it bounds more. The action for the 4sphere is negative and therefore one might think that the extremum encompassing more 4 -sphere should dominate. One must remember, however, that because of the conformal rotation the contour of $a$ integration is in the imaginary direction in the immediate vicinity of the extremum. Extrema of analytic functions are saddle points so that a maximum in a real direction is a minimum in an imaginary direction. The stationary configuration which contributes to the steepest descent evaluation of (4.56) is the one which is a maximum of the action in real directions and a least action configuration in imaginary directions. The extremum corresponding to the smaller part of the 4-sphere, therefore, provides the steepest descent approximation to the wave function. In fact, 


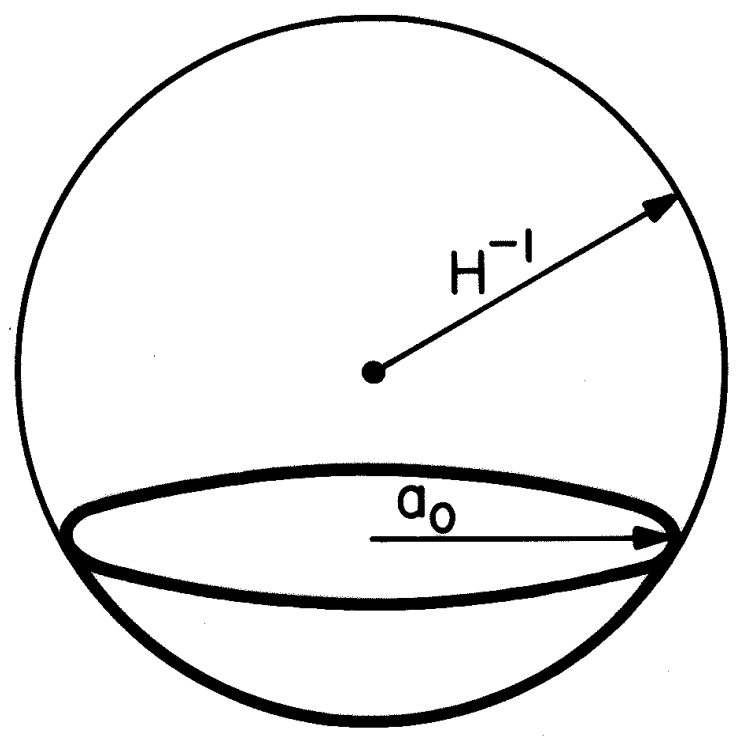

Figure 5: The real Euclidean extrema of the homogeneous, isotropic minisuperspace model with conformally invariant scalar field have the geometry of a 4-sphere of radius $H^{-1}$. The extremizing configuration which gives the semiclassical approximation to $\psi$ at $a_{0}<1 / H$ is a part of the 4 -sphere with a single 3 -sphere boundary of radius $a_{0}$. There are two possibilities corresponding to more than a hemisphere or less. The Euclidean functional integral prescription for $\psi$ identifies the smaller part of the 4-sphere as the contributing extremum. For $a_{0}>H^{-1}$ there are no real extrema. The orientation of the 3 -sphere in the 4 -sphere is arbitrary. The semiclassical vacuum of the matter field is thus de Sitter invariant.

the contour cannot be distorted to pass through the other extremum. We thus have for $a_{0}<1 / H$

$$
\psi\left(a_{0}\right) \approx N\left[-1+a_{0}^{2}-H^{2} a_{0}^{4}\right]^{-1 / 4} \exp \left[-\frac{1}{3 H^{2}}\left(1-H^{2} a_{0}^{2}\right)^{3 / 2}\right]
$$

where $N$ is an arbitrary normalizing factor.

If $a_{0}$ is increased to a value larger than $1 / H$ there are no longer any real extrema because a 3 -sphere of radius $a_{0}>1 / H$ cannot fit into a 4 -sphere of radius $1 / H$. There are, however, complex extrema. These can be obtained by changing $\tau \rightarrow \pm i t$ in Equation (4.58) so they solve

$$
\left(\frac{\dot{a}}{a}\right)^{2}=H^{2}-\frac{1}{a^{2}}
$$

An extremum is then a solution of the Lorentzian Einstein equations with positive cosmological constant. This solution is called de Sitter space. These complex extrema must contribute in complex conjugate pairs so that the wave function is real. By a standard WKB matching analysis we can establish the form of the wave function for 


$$
a_{0}>H^{-1}
$$

$$
\psi\left(a_{0}\right) \approx 2 N\left[H^{2} a_{0}^{4}-a_{0}^{2}+1\right]^{-1 / 4} \cos \left[\frac{\left(H^{2} a_{0}^{2}-1\right)^{3 / 2}}{3 H^{2}}-\frac{\pi}{4}\right] .
$$

This form could be derived by carefully following the extremum configuration as $a_{0}$ is increased along the heavy curve shown in Fig. 4.

In the region $a_{0}>H^{-1}$ the steepest descents approximation to the wave function is a real linear combination of solutions of the semiclassical form (4.33). In this region we find the geometric correlations of classical de Sitter space. The state of the matter field can be read of (4.35), (4.40) and (4.56). It is, up to normalization,

$$
\chi_{0}\left[\tau, \varphi_{0}^{(n)}\right]=\prod_{n} \exp \left[-\frac{1}{2} \omega_{(n)}\left(a(\tau) \varphi_{0}^{(n)}\right)^{2}\right] .
$$

In the minisuperspace model, the prescription for the wave function of the universe of Section 3.3 predicts this particular quantum state of matter in the limit of classical geometry and quantum field theory in curved spacetime.

The state specified in the field representation by (4.62) is familiarly known as the Euclidean de Sitter invariant vacuum state. To see this we note that in terms of the variables $\chi_{0}^{(n)}$ the wave function (4.62) is that of a field in its ground state in the Einstein static universe. In Fock space the state is therefore annihilated by the modes which are positive frequency in the conformal time $\eta$. In terms of $\varphi$ these are modes proportional to

$$
[a(\eta)]^{-1} \exp \left(-i \omega_{(n)} \eta\right) Y_{(n)}(\mathbf{x})
$$

This is the conventional definition of the Euclidean, de Sitter invariant vacuum [26].

As argued by D'Eath and Halliwell [23] the de Sitter invariance of the quantum state of the matter is an inevitable consequence of the symmetry of the Euclidean sum over histories prescription. In the defining region $a_{0}<H^{-1}$ the extremizing configuration which supplies the steepest descents approximation is the smallest part of a 4 -sphere bounded by a 3 -sphere of radius $a_{0}$. However, the wave function does not depend on the orientation of the 3 -sphere in the 4 -sphere. There is thus an $O(5)$ invariance which in the Lorentzian region corresponds to the de Sitter group.

Preparation of these lectures was supported in part by the National Science Foundation under Grant PHY 85-06686.

\section{A Problems}

1. Practice expressing cosmological and local observations in terms of correlations in the wave function of the universe. Are there observations which cannot be so expressed?

2. Provide a careful derivation of the Wheeler-DeWitt equation from the sum over histories which shows the connection between the factor ordering and the measure. Hint: See Ref. 28. 
3. Is there a complex contour in the space of 4-geometries and matter field configurations along which the sum over histories for quantum cosmology is convergent?

4. Discuss the regularization of quantum field theory in curved spacetime in the context of the semiclassical limit of quantum cosmology described in Section 4. Hint: See Ref. 23.

\section{B Notation}

For the most part we follow the conventions of Ref. 29 with respect to signature, curvature and indices. In particular:

Signature: $(-,+,+,+)$ for Lorentzian spacetimes. $(+,+,+,+)$ for Euclidean spacetimes. Indices: Greek indices range over spacetime from 0 to 3. Latin indices range over space from 1 to 3 .

Units: We use units in which $\hbar=c=1$. The Planck length is $\ell=(16 \pi G)^{\frac{1}{2}}=1.15 \times 10^{-32}$ $\mathrm{cm}$.

Covariant Derivatives: $\nabla_{\alpha}$ denotes a spacetime covariant derivative and $D_{i}$ a spatial one.

Traces and Determinants: Traces of second rank tensors $K_{\alpha \beta}$ are written as $K=K_{\alpha}^{\alpha}$ except when the tensor is the metric in which case $g$ is the determinant of $g_{\alpha \beta}$ and $h$ the determinant of $h_{i j}$.

Extrinsic Curvatures: If $n_{\alpha}$ is the unit normal to a spacelike hypersurface in either a Euclidean or Lorentzian spacetime, we define its extrinsic curvature to be

$$
K_{i j}=\nabla_{i} n_{j}
$$

Intrinsic Curvatures: Intrinsic curvatures are defined so that the scalar curvature of a sphere is positive.

Metric on the unit $n$-sphere: This is denoted by $d \Omega_{n}^{2}$ and in standard polar angles is

$$
\begin{array}{cc}
d \Omega_{2}^{2}=d \theta^{2}+\sin ^{2} \theta d \varphi^{2} & n=2 \\
d \Omega_{3}^{2}=d \chi^{2}+\sin ^{2} \chi d \Omega_{2}^{2} & n=3
\end{array}
$$

\section{References}

1. Discussions of the general problems encountered in the search for initial conditions may be found in R. Penrose, in General Relativity: an Einstein Centenary Survey, ed. by S. W. Hawking and W. Israel (Cambridge University Press, Cambridge, 1979), J. B. Hartle in Inner Space/Outerspace: the Interface between Cosmology and Particle Physics, ed. by E. W. Kolb, et.al. (University of Chicago Press, Chicago, 1986) and J. Barrow and F. Tipler, The Anthropic Principle, (Clarendon Press, Oxford, 1986). A sample of specific proposals for laws for initial conditions may be found in the article by Penrose cited above and in Refs 2-5. 
2. S. W. Hawking, in Astrophysical Cosmology: Proceedings of the Study Week on Cosmology and Fundamental Physics ed. by H. A. Brüch, G. V. Coyne and M. S. Longair (Pontificiae Academiae Scientiarum Scripta Varia, Vatican City, 1982), Nucl. Phys., B239, 257, 1984 and J. B. Hartle and S. W. Hawking, Phys. Rev., D28, 2960, 1983.

3. A. Vilenkin, Phys. Lett., B117, 25, 1983, Phys. Rev., D27, 2848, 1983, Phys. Rev., D30, 509, 1984, Phys. Rev., D32, 2511, 1985, TUTP preprint 85-7.

4. J. V. Narlikar and T. Padmanabhan, Physics Reports, 100, 151, 1983, T. Padmanabhan "Quantum Cosmology - The Story So Far" (unpublished lecture notes).

5. W. Fischler, B. Ratra, L. Susskind, Nucl. Phys. B, 259, 730, 1985.

6. The author's lectures at Cargese were somewhat more extensive than presented here. Some of this material can be found in the author's lectures in High Energy Physics 1985: Proceedings of the Yale Theoretical Advanced Study Institute ed. by M. J. Bowick and F. Gürsey (World Scientific, Singapore 1985).

7. For a clear review of the basic ideas in canonical quantum gravity in greater detail than can be presented here see K. Kuchar, in Relativity Astrophysics and Cosmology ed. by W. Israel (D. Reidel, Dordrecht, 1973) and in Quantum Gravity 2 ed. by C. Isham, R. Penrose and D. Sciama (Clarendon Press, Oxford, 1981).

8. See, e.g., H. Everett, Rev. Mod. Phys., 29, 454, 1957, the many articles reprinted and cited in The Many Worlds Interpretation of Quantum Mechanics, ed. by B. DeWitt, and N. Graham, (Princeton University Press, Princeton, 1973), M. Gell-Mann (unpublished), and the lucid discussion in R. Geroch, Noûs, 18, 617, 1984.

9. D. Finkelstein, Trans. N.Y. Acad. Sci., 25, 621, 1963; N. Graham, unpublished Ph.D. dissertation, University of North Carolina 1968 and in The Many Worlds Interpretation of Quantum Mechanics, ed. by B. DeWitt, and N. Graham (Princeton University Press, Princeton, 1973); and J. B. Hartle Am. J. Phys., 36, 704, 1968.

10. For a very clear discussion of this equivalence and its consequences see C. M. Caves, Phys. Rev., D33, 1643, 1986 and ibid, D35, 1815, 1987.

11. H. Salecker and E. P. Wigner, Phys. Rev., 109, 571, 1958.

12. B. DeWitt, Phys. Rev., 160, 1113, 1967.

13. A. Peres, Am. J. Phys., 48, 552, 1980.

14. D. Page and W. Wooters, Phys. Rev., D27, 2885, 1983, W. Wooters, Int. J. Th. Phys., 23, 701, 1984. 
15. See, e.g., J. B. Hartle and S. W. Hawking, Phys. Rev., D28, 2960, 1983, S. W. Hawking (to be published), D. N. Page (to be published). J. B. Hartle, Class. Quant. Grav., 2, 707, 1985, A. Anderson and B. DeWitt, Found. Phys., 16, 91, 1986.

16. See, e.g., H. Hamber and R. Williams, Phys. Lett., 157B, 368, 1985, Nucl. Phys., B267, 482, 1986, ibid, B269, 712, 1986; H. Hamber in Critical Phenomema, Random Systems and Gauge Theories: Les Houches 1984 ed. by R. Stora and K. Osterwalder (Elsiever Science Publishers, Amsterdam, 1986); J. B. Hartle, J. Math. Phys., 26, 804, 1985.

17. H. Leutwyler, Phys. Rev., 134, B1155, 1964, B. S. DeWitt, in Magic Without Magic: John Archibald Wheeler, ed. by J. Klauder, (Freeman, San Francisco, 1972), L. Faddeev, and V. Popov, Usp. Fiz. Nauk., 111, 427, 1973 Sov. Phys. -Usp., 16, 777, 1974, E. Fradkin and G. Vilkovisky, Phys. Rev., D8, 4241, 1973, M. Kaku, Phys. Rev., D15, 1019, 1977.

18. E.g., S. W. Hawking in Relativity Groups and Topology II ed. by B. DeWitt and R. Stora (Elsevier, Amsterdam, 1984).

19. G. Gibbons, S. W. Hawking and M. Perry, Nucl. Phys., B138, 141, 1978, J. B. Hartle and K. Schleich, The Conformal Rotation in Linearized Gravity in Quantum Field Theory and Quantum Statistics, ed. by I. A. Batalin, C. J. Isham and G. A. Vilkovisky), Adam Hilger, Bristol, 67-87, (1987), K. Schleich, Conformal Rotation in Perturbative Gravity, Phys. Rev D 36, 2342-2363 (1987).

20. J.A. Wheeler in Problemi dei fondamenti della fisica, Scoula internazionale di fisica "Enrico Fermi" Corso 52 ed. by G. Toraldo di Francia (North Holland, Amsterdam, 1979).

21. T. Banks, Nucl. Phys., B249, 332, 1985.

22. S. W. Hawking and J. Halliwell, Phys. Rev., D31, 1777, 1985.

23. P. D'Eath and J. Halliwell, Fermions in Quantum Cosmology, Phys. Rev. D, 35, 1100 (1987).

24. R. Brout, G. Horwitz and D. Weil, On the Onset of Time and Temperature in Cosmology, Phys. Lett B, 192, 318 (1987).

26. S. Hojman, K. Kuchar, and C. Teitelboim, Ann. Phys. (N.Y.), 76, 97, 1976.

27. See, e.g., N. D. Birrell and P. C. W. Davies Quantum Fields in Curved Space (Cambridge University Press, Cambridge, 1982).

28. A. Barvinsky and V. N. Ponomariov, Phys. Lett., 167B, 289, 1986, A. Barvinsky, Phys. Lett., 175B, 401, 1986.

29. C. Misner, K. Thorne, and J. A. Wheeler, Gravitation (W. H. Freeman, San Francisco, 1970). 